Proceedings

\title{
A Model of Deceitful Information Communication: Some Views on Theory and Practice of Semantic Information $^{\dagger}$
}

\author{
Nan Wang and Bocong Li * \\ School of Humanities and Social Sciences, University of Chinese Academy of Sciences, 100049 Beijing, China; \\ wangnan@ucas.ac.cn \\ * Correspondence: libocong@ucas.ac.cn; Tel.: +86-10-88256360 \\ + Presented at the IS4SI 2017 Summit DIGITALISATION FOR A SUSTAINABLE SOCIETY, Gothenburg, \\ Sweden, 12-16 June 2017.
}

Published: 9 June 2017

\begin{abstract}
This presentation is going to propose a model of deceitful information communication in terms of the Shannon-Weaver model, which is a classical model in theory of communication. It also makes a brief analysis on the four characteristics of the new model.
\end{abstract}

Keywords: model; deceitful information; communication; semantic information

\section{Introduction}

Recently, many telecommunication fraud cases have been reported in China, which is a new way to swindle unsuspecting victims.

As a new way of fraud, telecommunication fraud resulted in serious harms and caused a social shock. Media reported that some swindling gangs were well organized and the fraud process was carefully planned.

Some scholars analyzed telecommunications fraud cases from the legal point of view, other scholars from the ethical point of view. Now, we'll analyze them from the point of view of semantic information and communication. Telecommunications fraud cases afford a new clue to the puzzle of what semantic information means.

\section{Two Kinds of Semantic Information and Two Kinds of Communication}

1948 is a historic year. That year saw the publication of A Mathematical Theory of Communication by Claude Elwood Shannon and of Cybernetics: Or Control and Communication in the Animal and the Machine by Norbert Wiener. It should be noted that the word communication is included in the title of the two book, which means that both Shannon and Wiener paid attention to communication.

Wiener is regarded as the originator of cybernetics and Shannon as the originator of information theory. While Shannon researched information with a mathematical approach, Wiener researched control with a comprehensive approach. Wiener put forward a famous thesis: information is information, not matter or energy [1], which is a philosophical thesis in essence. Since then, information has become a frequently used word and a basic category in science, technology, engineering, and society.

What is information? What is communication? These two questions are closely related and entangled with each other.

As to information, Prof. Yixin Zhong put forward a comprehensive explanation recently. "The information must be the trinity of its three components: the form termed syntactic information, the meaning termed semantic information, and the utility termed pragmatic information" [2]. 
As to communication, Warren Weaver put forward a comprehensive explanation in 1949. He thought the communication can be divided into three levels [3]. Specifically, level A is how accurately the symbols of communication can be transmitted, which is a technical problem in essence. Level B is how precisely the transmitted symbols convey the desired meaning, which is a semantic problem in essence. Level $\mathrm{C}$ is how effectively the received meaning affects conduct in the desired way, which is an effectiveness problem in essence.

It is easy to see the similarities between the three levels of communication and the three components of information. To a certain extent, syntactic information is associated with level A of communication, semantic information with level B, and pragmatic information with level C.

As the originator of information theory, Shannon distinguished signal from message, which suggested that Shannon realized that communication concerns both syntax and semantics. Despite this, Shannon still held, "The fundamental problem of communication is that of reproducing at one point either exactly or approximately a message selected at another point. Frequently the messages have meaning; that is they refer to or are correlated according to some system with certain physical or conceptual entities. These semantic aspects of communication are irrelevant to the engineering problem." [4], p. 379 Obviously, Shannon excluded the semantic dimension in the field of (technical) communication.

Due to Shannon's influence, the study of semantic information has been excluded in the field of information science and engineering to a large extent for a long time. It should be admitted that this is not accidental. For example, it is impossible to imagine that in the age of telegraph communication a telegram can be charged by semantic information rather than by words. Despite all this, while few engineers and scholars attached importance to the study of semantic problems in the field of information science and technology, some scholars attached importance to the study of semantic problems in the field of linguistics and philosophy. We have to admit that semantic information and semantic information communication is one of the most difficult issues.

Recently, the situation began to change. Since the beginning of 21st century, some scholars, such as Luciano Floridi and Yixin Zhong have placed great emphasis on the importance of semantic information. Semantic information involves many problems. This presentation is merely going to discuss a particular issue: deceitful information and deceitful communication.

As to the definition of semantic information, many scholars regard semantic information as meaningful and well-formed data only if they also are qualified as truthful. They do not think of false semantic information as a type of semantic information. In their studies, the research of false semantic information is excluded.

However, in English, the word "false" has two different meanings. One refers to something incorrect, untrue, or mistaken. Another refers to something fabricated in order to deceive. The former is an epistemological and logical topic in essence, and the latter is an ethical and legal topic in essence. So there are two kinds of false semantic information, and two kinds of false communication.

Till now, in the field of information science and technology, the false semantic information that engineers and scientists mentioned is the first kind, i.e., the epistemologically and logically false semantic information, but not the second kind, i.e., the ethically and legally false semantic information.

From the academic perspective, telecom fraud cases suggest that it is impossible to continue to ignore study of the second kind of false semantic information. However, in this presentation, to a certain extent, the second kind of false semantic information will be regarded mainly as an epistemological topic instead of an ethical and legal one from a new perspective.

At present, when considering semantic information, philosophers and information scientists have only concerned about true semantic information and completely ignore the false information problem.

Now, in the field of information ecology, the second kind of false information can be not only an ethical and legal issue but also an epistemological and informational one. From the point of view of information ecology, semantic information can be divided into two kinds: normal semantic information and deceitful semantic information. Correspondingly, communication can be divided into two kinds: normal communication and deceitful communication. 


\section{A Simple Model of Deceitful Information Communication}

Cheating is a common phenomenon in society. The reasons for cheating are various. Some cases of deceit are criminal and others are called white lies. The process of deceitful communication is different from that of normal communication.

As to normal communication, Shannon-Weaver Model was put forward, which has been called the "mother of all models." [5]. Shannon-Weaver model can be illustrated as follows (Figure 1).

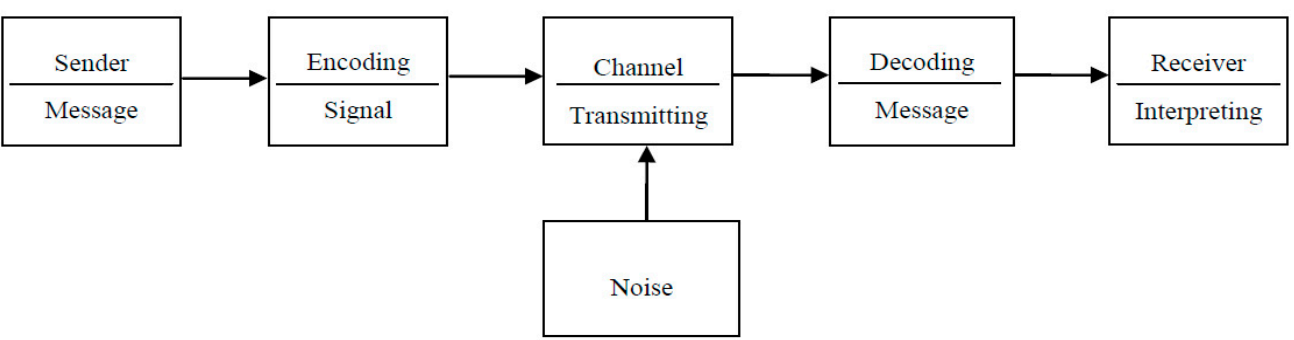

Figure1. The Shannon-Weaver Model

On one hand Shannon distinguished signal from message; on the other hand Shannon says, "The fundamental problem of communication is that of reproducing at one point either exactly or approximately a message selected at another point. Frequently the messages have meaning; that is they refer to or are correlated according to some system with certain physical or conceptual entities. These semantic aspects of communication are irrelevant to the engineering problem" [4]. It means that Shannon excluded the semantic dimension in the field of communication. So this model deals with various concepts like information source, transmitter, noise, channel, message, receiver, channel, information destination, encode and decode.

For deceitful communication, a new model should be proposed, because Shannon-Weaver model no longer fits for its situation. A model of deceitful communication, which is derived from Shannon-Weaver model, can be illustrated as follows (Figure 2).

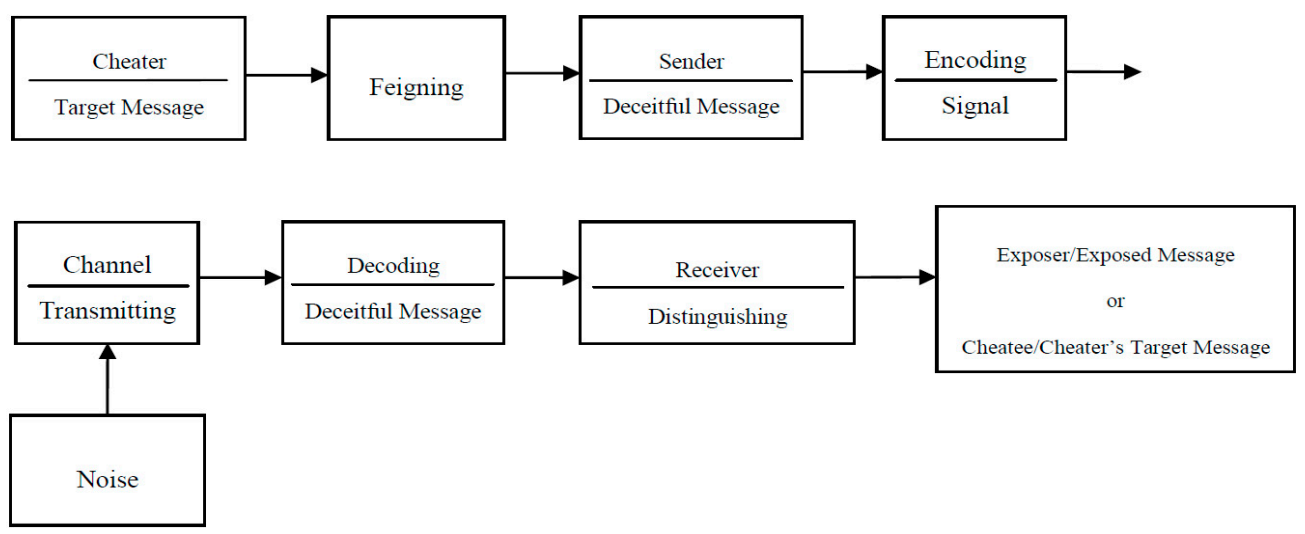

Figure2. The model of Deceitful Communication

This model deals with some new concepts, like semantic information, cheater, cheatee, target message, feigning, deceitful message, which distinguish false semantics from true semantics. 


\section{Characteristics of Deceitful Communication}

In comparison to the Shannon-Weaver model, the model of deceitful communication has the following characteristics.

First, as to the role of structure of communication in this model, the sender of deceitful semantic information is not a normal sender but a cheater, and the receiver of deceitful semantic information can become a cheatee or an exposer according to whether he can distinguish the deceitful information from normal information.

Second, as to the nature of semantic information in this model, the semantic information communicated is deceitful semantic information but not normal semantic information.

Third, as to operations in the communication process, while Shannon-Weaver model involves mainly encoding and decoding, the model of deceitful semantic information communication involves feigning and distinguishing, in addition to encoding and decoding.

Forth, as an information processing method, feigning and distinguishing semantic information are much more complex than encoding and decoding in the Shannon-Weaver model. Encoding and decoding mainly use technical methods, while feigning and distinguishing use psychological and social methods.

Fifth, in terms of forms of signal, a kind of signal will be changed into another kind of signal by encoding. However, the form of signal will not be changed by feigning. More exactly, in deceitful communication a deceiver has to use the same syntax and lexicon that the deceiver uses.

Sixth, in the process of deceitful communication, if the receiver cannot distinguish the deceitful message successfully, he will become a cheatee and the cheater will get the purpose. If the receiver can distinguish the deceitful message successfully, he will become an exposer and the cheating will fail.

Last but not least, in the aspect of ethics and axiology, the concept deceitful information can be interpreted as a neutral concept rather than a merely negative one. For examples, when one tells a white lie or a spy for the sake of justice sends deceitful information in order to deceive the enemy, this sort of deceitful information has positive value. All in all, the impact of deceitful information can be negative or positive.

\section{Deception as Both an Old and New Issue}

Deception is a common social phenomenon, which is both an old and new problem in society. In the academic circle, deception has been regarded mainly as an ethical problem for a long time, which means that it is an old issue. Now, when we study semantic information and information ecology, deceitful information communication becomes a new issue.

In the model of deceitful communication, distinguishing the deceitful semantic information is very complex and very difficult. Till now, to deceitful communication, there is no in-depth analysis and research on the problem in theory, and in practice, there is no better solution to the deceitful communication problem. In this presentation, a model of deceitful communication is put forward. The aim of the model is not to give an answer, but merely to give a clue.

In the Shannon-Weaver model, external noise makes trouble, while in the model of deceitful communication, internal feigning makes trouble. The former is a technical problem in essence and the latter a complex social and psychological semantic problem. In other word, in the Shannon-Weaver model there is only noise but not deception.

Conflicts of Interest: The authors declare no conflict of interest.

\section{References}

1. Wiener, N. Cybernetics: Or Control and Communication in the Animal and the Machine; The MIT Press: Cambridge, MA, USA, 1948.

2. Zhong, Y.X. A theory of semantic information. China Commun. 2017, 14, 1-17.

3. Warren, W. Recent Contributions to the Mathematical Theory of Communication. In ETC: A Review of General Semantics; Available online: http://www.panarchy.org/weaver/communication.html (accessed on 9 June 2017). 
4. Shannon, C.E. A Mathematical Theory of Communication. Bell Syst. Tech. J. 1948, 27, 379-423, $623-656$.

5. Anonymous. Available online: https://en.wikipedia.org/wiki/Shannon-Weaver_model\#cite_ref-4 (accessed on 9 June 2017).

(C) (

(C) 2017 by the authors. Licensee MDPI, Basel, Switzerland. This article is an open access article distributed under the terms and conditions of the Creative Commons Attribution (CC BY) license (http://creativecommons.org/licenses/by/4.0/). 